\title{
MALGORZATA KOLANKOWSKA
}

ORCID: 0000-0002-9804-9210

\section{Violencia contra la mujer a través de los testimonios de las crónicas periodísticas}

\author{
Palabras clave: violencia - mujer - periodismo - feminicidio - machismo \\ - crónica.
}

\section{Resumen}

El artículo presenta el problema de la violencia contra la mujer en los medios hispanos contemporáneos. El estudio describe el concepto y la forma en que es abordado por el periodismo. Demuestra que los periodistas eligen la investigación personal y el género de la crónica periodística para mostrar el dolor, el sufrimiento y la muerte de las mujeres en diferentes contextos sociales. Su trabajo es a menudo la única manera de dar un testimonio sobre los problemas silenciados.

\section{Introducción}

El tema de la violencia contra la mujer resurgió a finales del año 2019 como tema de interés en los medios de comunicación, como una voz más del denominado movimiento $\mathrm{Me}$ Too, con la confesión de la escritora Elena Poniatowska de que Juan José Arreola abusó de ella cuando tenía veintidós años ${ }^{1}$, lo cual describe de forma implícita en su último libro El amante polaco. La declaración de una mujer de ochenta y siete años ha tenido mucha repercusión en los medios de comunicación. La prensa, a través de artículos de opinión, ha destacado muchos de los aspectos sociales o jurídicos de lo que suponen esas discusiones, y analizarlas permite conocer tanto cómo afrontan los medios de comunicación el tema de la violencia de género, como a través de qué formatos o géneros informativos se genera opinión entre los públicos. La escritora

1 Associated Press, "Elena Poniatowska se suma al \#Me too; hace fuertes confesiones", El Excesior, 16.12.2019, <https://www.excelsior.com.mx/expresiones/elena-poniatowska-se-suma-almetoo-hace-fuertes-confesiones/1353486>, 29 de diciembre de 2019. 
Carme Riera alude a la confesión de Elena Poniatowska en su columna titulada "Violencia de género"2 en la que pone hincapié en el hecho de que el número de víctimas de la violencia sigue siendo muy alto en España y lo vincula con el patriarcado construido en la relación del poder y el sentido de superioridad masculina respecto a las féminas. En su opinión, la confesión de Poniatowska abre paso a un cambio social tanto en España como en Hispanoamérica.

\section{Objetivo y metodología}

El objetivo del presente ensayo es analizar la violencia contra la mujer en los medios de comunicación y para demostrarlo se han escogido los ejemplos en que el problema ha sido abordado desde distintas perspectivas, siendo la crónica el género más destacado. Primero se define el concepto y su relación con el mundo mediático y a continuación se citan los ejemplos que reflejan la importancia del problema en los textos escritos, películas documentales y la fotografía. La selección de las crónicas se basa en los tres elementos básicos del periodismo: la palabra, el sonido y la imagen. Siguiendo la idea de Ryszard Kapuściński, se documenta que cada uno de los periodistas mencionados a continuación se guía por los cinco sentidos: estar, ver, oír, compartir y pensar³

\section{El concepto de la violencia contra la mujer}

En primer lugar, hay que aproximarse al concepto de la violencia contra la mujer así como su relación y visibilidad en los medios de comunicación como un asunto de interés informativo.

Según Antonio Jesús Yugueros García ${ }^{4}$, el primer documento en que encontramos una clara definición del concepto de la violencia es la Declaración sobre la Eliminación de la Violencia contra la Mujer:

por "violencia contra la mujer" se entiende todo acto de violencia basado en la pertenencia al sexo femenino que tenga o pueda tener como resultado un daño o sufrimiento físico, sexual o sicológico para la mujer, así como las amenazas de tales actos, la coacción o la privación arbitraria de la libertad, tanto si se producen en la vida pública como en la vida privada 5 .

2 C. Riera, "Violencia de género", La Vanguardia, 22.12.2019, <https://www.lavanguardia. com/opinion/20191222/472400291222/violencia-de-genero.html>, 29 de diciembre de 2019.

${ }_{3}$ R. Kapuściński, Los cinco sentidos de un periodista: (estar, ver, oír, compartir y pensar), México, Fondo de Cultura Económica, 2003, consultado en: <https://reportepolitico.files.wordpress.com/2011/03/kapuscinski_los_cinco_sentidos_del_periodista.pdf >, 15 de mayo de 2020 .

4 A. Jesús Yugueros García, "La violencia contra las mujeres: conceptos y causas", Barataria Revista Castellano-Manchega de Ciencias Sociales, 18, p. 148.

5 ONU, "Declaración sobre la Eliminación de la Violencia contra la Mujer", aprobada en Resolución de la Asamblea General de Naciones Unidas 48/104, del 20 de diciembre de 1993, 
En 2006 las Naciones Unidas subrayan que la violencia contra la mujer es "una forma de discriminación y una violación de los derechos humanos"6. Yugueros García resalta que los términos "violencia de género" y "violencia contra las mujeres" adquieren un carácter sinónimo, al contrario del concepto de "la violencia doméstica". Por otro lado, Leticia Casique Casique y Antonia Regina Ferreira Furegato ${ }^{7}$ afirman que la violencia está relacionada con la percepción biológica y social del sexo.

Según los datos proporcionados por el Instituto Oficial de Radio y Televisión $\mathrm{RTVE}^{8}$ el tema de la violencia doméstica (como foco de interés para la agenda mediática) surge en los medios de comunicación en 1997 con el caso de Ana Orantes, que había denunciado malos tratos en un programa de televisión a través de una entrevista y poco después fue asesinada por su marido, lo cual abrió la caja de Pandora.

Yugueros García9, aludiendo a los estudios de Nogueiras y Melero, además del silencio, menciona la naturalización y la invisibilidad de la violencia cuyo efecto era, sobre todo, la falta de conciencia por parte de las víctimas y la sociedad, resalta que "las mujeres son violentadas por el simple hecho de ser mujeres, no hay ninguna otra motivación, como ocurre en las demás formas de violencia"10.

La Secretaría de las Naciones Unidas ${ }^{11}$ afirma que hay varias formas de violencia: física, sexual, psicológica y económica, mientras que Casique Casique y Ferreira Furegato ${ }^{12}$ dinstiguen: agresión física, agresión psicológica y social.

Las definiciones más recientes las encontramos en el estudio de M. Pilar Matud, Demelza Fortes, Esther Torrado y Saray Fortes ${ }^{13}$ en que se resalta la importancia de la actitud frente al problema de la violencia: "para comprender y erradicar la violencia contra las mujeres, así como para llevar a cabo inter-

publicada el 23 de febrero de 1994, en: <http://www.ohchr.org/SP/ProfessionalInterest/Pages/ViolenceAgainstWomen.aspx>.

6 "Fin a la violencia contra la mujer: Hechos, no palabras. Informe del Secretario General", 2006, <https://www.un.org/es/events/endviolenceday/2006/pdf/v.a.w-exeS-use.pdf>.

7 L. Casique Casique, A.R. Ferreira Furegato, "Violencia contra mujeres: reflexiones teóricas", Revista Latino-americana de Enfermagem, Ribeirão Preto, vol. 14, no. 6, Nov./Dec. 2006, $<$ http://www.scielo.br/scielo.php?pid=s0104-11692006000600018\&script=sci_arttext\&tlng=es $>$, 29 de diciembre de 2019.

8 Instituto Oficial de Radio y Televisión. RTVE, Mujer, violencia y medios de comunicación, Madrid, 2002, p. 7.

9 A. Jesús Yugueros García, op. cit., p. 150.

10 Ibidem, pp. 150-151.

${ }^{11}$ UN Department of Public Information, DPI/2546C, Noviembre 2009, "Violencia contra las mujeres", disponible en línea.

${ }^{12}$ L. Casique Casique, A.R. Ferreira Furegato, op. cit.

13 M. Pilar Matud, D. Fortes, E. Torrado, S. Fortes, Violencia contra las mujeres, La Laguna 2018. 
venciones con víctimas y agresores, diseñar y ejecutar programas y estrategias de prevención es imprescindible seguir una perspectiva de género"14.

\section{La relación entre los medios de comunicación y la violencia}

May Silva Ortega ${ }^{15}$ opina que los medios de comunicación constituyen una de las principales herramientas que pueden desempeñar la función educativa en relación con la erradicación de la violencia de género e indica que uno de sus fines más importantes es la sensibilización y promoción de "acciones positivas"16. Miguel Lorente Acosta ${ }^{17}$ comparte la misma opinión, no obstante, presta atención a los peligros relacionados con la actuación de los medios, ya que todo depende de la actitud que toman a la hora de presentar noticias. El autor distingue dos posturas: la imparcialidad y la neutralidad.

Omar Rincón ${ }^{18}$, periodista y crítico de televisión colombiano, afirma que en América Latina la voz de la mujer sigue pasando desapercibida, por lo cual existe la necesidad de repensar su presencia en los medios de comunicación y propone un decálogo del "periodismo en perspectiva de género", en que subraya la importancia de una "narrativa testimonial" que sale desde la óptica de lo emocional que además propone algunas soluciones y alternativas.

Concluyendo, lo que propone Rincón es el periodismo lento ${ }^{19}$, que no forma parte de las agendas oficiales y siempre se enfoca en el análisis de los hechos. El periodismo slow es una respuesta a la rapidez y superficialidad de los medios de comunicación y es uno de los enfoques que se centran en el análisis del problema y reflejan desde qué cuerpo contar las historias. Ese trabajo requiere tiempo, a veces semanas o, incluso, meses de trabajo, de investigación. A menudo se utiliza el formato multimedia, es decir, se une el texto con las fotos, el video o el podcast.

14 Ibidem, p. 19

15 M. Silva Ortega, "El papel de la educación y los medios de comunicación en la errdicación de la violencia contra las mujeres", en: M. del Mar Ramírez Alvarado (coord.), Medios de comunicación y violencia contra las mujeres, Sevilla, Instituto Andaluz de la Mujer, Fundación Audiovisual de Andalucía, 2003, pp. 24-25.

16 Ibidem, p. 30.

17 M. Lorente-Acosta, "La violencia contra las mujeres en los medios de comunicación: entre la noticia y la información", en: M. del Mar Ramírez Alvarado (coord.), op. cit., pp. 34-35.

18 O. Rincón, "La narrativa en perspectiva de género", en: S. Santoro, ;Sin nosotras se les acaba la fiesta! [América Latina en perspectiva de género], Bogotá, Centro de Competencia en Comunicación para América Latina, Friedrich Ebert Stiftung Artemisa Comunicación, 2009, pp. $7-8$.

19 Véase: B. Köhler, D. Sabria, J. Blumtritt, "The Slow Media Manifesto", en: $<$ http://en.slow-media.net/manifesto>; G. Rosique-Cedillo, A. Barranquero-Carretero, "Periodismo lento (slow journalism) en la era de la inmediatez. Experiencias en Iberoamérica", El Profesional de la Informacion, 24 (4):451, July 2015. 
Un análisis de una selección de contenidos de la prensa permitirá aproximarse a la idea de la violencia contra la mujer a través de los medios de comunicación. Como es una aproximación a la problemática, nos centramos en cómo se presentan las víctimas y cómo se sensibiliza y educa a la sociedad por medio de dichas historias.

\section{La violencia presentada en una crónica periodística}

Uno de los géneros que permiten un mayor acercamiento a los hechos y una perspectiva más personal es la crónica, que, como señala Carmen Rodríguez Wangüemert ${ }^{20}$, se ha hecho sumamente importante en los últimos años: "En las crónicas periodísticas un elemento de gran valor es la consideración de la visión personal que aporta el narrador que permite al medio diferenciar su oferta informativa de la de otros medios. Ese valor testimonial realiza una forma de interpretar la realidad del propio medio".

El proyecto realizado por María Rigat, Omar Rinón y Sonia Santoro reúne las crónicas de los quince países en Latinoamérica. Cada texto está escrito por una mujer y concierne a los problemas de las féminas. Todas las autoras utilizan las crónicas porque son las formas periodísticas que permiten enfatizar la perspectiva de las protagonistas. Como resaltan las cronistas su idea era "difundir otra manera de hacer periodismo: que mire las realidades haciendo foco en las diferencias y desigualdades persistentes entre varones y mujeres - atravesado por las distintas etnias, orientaciones sexuales, edades-, por las que las mujeres siguen siendo discriminadas" 21 . Lo más importante era escribir desde el cuerpo de una mujer y presentar un panorama de diversos problemas que atañen a las latinoamericanas: el narcotráfico, la prostitución, el abuso, la injusticia, el maltrato, etc. En el libro se encuentran los textos que demuestran diferentes niveles de discriminación, por ejemplo, en la calle o a nivel racial como es el caso de las indígenas en Bolivia o las negras en Uruguay. Merece una especial atención el texto de Zoraida Portillo ${ }^{22}$ que aborda el tema de la violencia simbólica en la prensa amarilla peruana en que las mujeres son presentadas como víctimas o malas, pero siempre dependientes de los hombres. La autora presta atención a la influencia negativa que tiene la objetivación del cuerpo femenino, lo cual puede llevar a la conclusión de que sea "un ser incompleto si no tiene un hombre al lado" 23 . Además, Portillo indica otro aspecto

20 C. Rodríguez Wangüemert, "Las crónicas: algunas ideas sobre la credibilidad en el periodismo interpretativo", Estudios sobre el mensaje periodístico, 2005, pp. 171-172.

21 S. Santoro, "Escribir lo que se nos de la gana", en: S. Santoro (ed.), op. cit., p. 12.

22 Zoraida Portillo, "Prensa chicha en Perú. Cuando las noticias se farandulizan", en: S. Santoro (ed.), ;Sin nosotras..., op. cit., pp. 65-74.

${ }^{23}$ Ibidem, p. 69. 
negativo de la prensa chicha, la visión trastornada del feminicidio, tratado más bien como un castigo merecido que un crimen.

El panorama lo cierra la crónica de Nora Patricia Jara López ${ }^{24}$ sobre las muertas y desaparecidas de Juárez. Aquí cabe citar a algunos autores que han dedicado mucho espacio al tema de feminicidio, como Victor Ronquillo ${ }^{25}$, Fabrizio Mejía Madrid ${ }^{26}$ y Guadalupe Lizárraga ${ }^{27}$. El primero en abordar el tema fue Ronquillo, que reconstruye la historia de Ciudad Juárez desde el año 1993 cuando comenzó el horror. Es un libro en que el autor se identifica con las víctimas. Su forma de narrar es muy particular, utiliza el lenguaje literario, a veces metáforas, como en el caso de la descripción de las cruces que "hablan de la tragedia" y "son un triste monumento a la impunidad" 28 . Ronquillo no se mantiene imparcial, vuelve a repetir la acusación una y otra vez a lo largo del libro, demuestra la indolencia del gobierno y de la policía, busca a los responsables del crímen.

En tales circunstancias destaca la labor de la periodista mexicana de Los Angeles Press, Guadalupe Lizárraga, que se ve obligada a tomar riendas y en respuesta a una solicitud de ayuda en Facebook, empieza una investigación. La reportera defiende que el periodismo en México está infectado con "amnesia macondiana" 29 , lo cual va vinculado con "la incertidumbre y la violencia normalizada" 30 en Latinoamérica causados por la militarización de los países oficialmente democráticos. En ese contexto Lizárraga decide a trabajar por cuenta propia, ya que en el periodismo independiente en la red encuentra la esperanza $^{31}$. Su libro Desaparecidas de la morgue es fruto de tres años de investigación sobre el feminicidio y la trata sexual en México. La reportera utiliza la crónica para relatar los hechos y su propia investigación en primera persona. Eso le da más credibilidad a la historia y resalta la responsabilidad de la misma por los descubrimientos. El libro contiene descripciones de la investigación, intercambio de correspondencia con las familias de las víctimas, fragmentos de las conversaciones teléfonicas y entrevistas. Es un ejemplo del periodismo empeñado en educar, sensibilizar y desmentir.

${ }^{24}$ N.P. Jara López, "Crímenes de odio en México. Las cruces de las mujeres de Juárez”, en: S. Santoro (ed.), iSin nosotras..., op. cit., pp. 149-157.

25 V. Ronquillo, Las muertas de Juárez: Crónica de una larga pesadilla, Madrid, Temas de hoy, 2004.

26 F. Mejía Madrid, “El teatro del crimen”, en: J. Carrión (ed.), Mejor que ficción. Crónicas ejemplares, pp. 265-279.

27 G. Lizárraga, Desaparecidas de la morgue, Editorial Casa Fuerte, Imperial Beach CA.

28 V. Ronquillo, op. cit., p. 52.

29 G. Lizárraga, "El periodismo independiente frente a la violencia en México", en: J.L. González-Esteban, C.M. López-Rico (eds.), Periodismo, derechos humanos, migración y fronteras. Vigencia y legado de Ryszard Kapuściński, Murcia, Diego Marín, 2017, p. 120.

30 Ibidem, p. 121.

31 Ibidem, p. 122. 


\section{Violencia ilustrada con un documental}

La violencia es una de las materias más delicadas para contar. Es una forma de contacto con el dolor y sufrimiento. Chelo Álvarez-Stehle, nacida en España, es periodista y su principal preocupación es el tema del maltrato de las mujeres. En 2017 estrenó su documental titulado Arenas de silencio: Olas de valor. Chelo Álvarez-Stehle en su trabajo demuestra la cadena de violencia entre niñas, adolescentes y mujeres maduras. Su propia historia que intercala los dramas de otras mujeres de distintas partes del mundo es el hilo conductor del documental y muestra la escala del fenómeno de la violencia contra el sexo femenino. El silencio se convierte en la valentía: "Uno de los rasgos del film es la necesidad de rasgar la densa capa de silencio que asfixia todo el abanico de aspectos de violencia sexual, desde incesto a abuso del clero, violación y trata" 32 .

\section{Violencia ilustrada con fotoperiodismo}

La fotografía siempre ha tenido mucha importancia en el periodismo, teniendo a menudo el valor de una prueba material. Uno de los periodistas españoles más destacados en esta materia es Gervasio Sánchez que ha trabajado como fotoperiodista en muchos conflictos armados. Su último proyecto titulado Violencias contra las mujeres en conflictos internacionales ${ }^{33}$ se estrenó en 2019 en el Centro de Historias de Zaragoza. Sánchez reúne fotos de distintos viajes para mostrar las repercusiones de conflictos armados en mujeres y niñas. Al hablar de su oficio ${ }^{34}$, opina que lo peor para las víctimas es el silencio: "ese silencio que hace más daño que el grito más agudo". Presenta doce historias de varios países. Cada foto es un pretexto para hablar de otro aspecto de violencia provocada por el conflicto bélico. A través de los retratos distorsionados las féminas recuperan la dignidad. Alison Landsberg dice que las fotos son como prótesis de la memoria ${ }^{35}$ : en el caso de las imágenes del periodista español son fotos que causan un dolor fantasma, un dolor que despierta la conciencia sobre la guerra.

32 Ibidem, p. 157.

33 G. Sánchez, "Violencias contra las mujeres", El Heraldo, 17.12.2019, <https://www.heraldo. es/ noticias/internacional/2019/12/17/violencias-contra-las-mujeres-exposicion-de-fotografias-degervasio-sanchez-1349321.html?fbclid=IwAR3iUzL_kZA5K4CzaDumknr-YSnQoPOwgYcI8AUDDQCLaKgvdURJ8nFn_cY>, 30 de diciembre de 2019.

34 G. Sánchez, "Guerras, mentiras y juegos de vídeo", en: M. Leguineche, G. Sánchez, Los ojos de la guerra, Barcelona, Debolsillo, 2001, p. 472.

35 A. Landsberg, Prosthetic Memory: The Transformation of American Remembrance in the Age of Mass Culture, New York, Columbia University Press, 2004. 
En esta línea van los proyectos coordinados por dos plataformas de periodismo independiente, Periodismo Humano ${ }^{36}$ y Revista $5 \mathrm{~W}^{37}$. Los dos sitios web reúnen diversos materiales en forma de crónicas, videos, fotorreportajes y podcasts que tratan sobre los temas de injusticia, violencia, inmigración, etc., en diferentes partes del mundo. En cada uno de ellos la violencia contra la mujer ocupa un lugar especial ${ }^{38}$.

\title{
8. Conclusiones
}

La idea del artículo ha sido presentar las formas de contar la violencia contra la mujer en los medios de comunicación. Se ha demostrado que el tema sigue vigente, sobre todo, en relación con los países de Tercer Mundo. Los académicos y los mismos periodistas ven la necesidad de relatar la violencia, educar y concienciar en oposición al desarrollo de la violencia simbólica promovida por la prensa amarilla y los videos que acompañan, por ejemplo, el reguetón y conllevan la neutralización de la violencia contra el sexo femenino. Los géneros que más se utilizan son la crónica y el reportaje, centrados en la presencia del periodista. A menudo se aprovechan las herramientas multimedia y las redes sociales con el objetivo de atraer la atención del público.

No cabe duda de que la violencia contra la mujer debe ser documentada y contada, ya que es un modo de sensibilizar tanto a los opresores como a las víctimas. Las mujeres se dan cuenta de que han sido víctimas, descubren las fuentes de su dolor interno. El periodismo actual debe luchar contra el silencio y animar a oponerse a la misoginia y a la violencia basada en el simple hecho de ser mujer.

\section{Referencias bibliográficas}

\author{
ASSOCIATED PRESS \\ 2019 "Elena Poniatowska se suma al \#Me too; hace fuertes confesiones", El Excelsior, \\ 16.12.2019, <https://www.excelsior.com.mx/expresiones/elena-poniatowska-se-suma-al- \\ metoo-hace-fuertes-confesiones/1353486>, 29 de diciembre de 2019. \\ ÁLVAREZ-STEHLE Chelo \\ 2017 “Sands of silence: waves of courage”, en: González-Esteban J.L., López-Rico C.M. (eds.), \\ Periodismo, derechos humanos, migración y fronteras. Vigencia y legado de Ryszard \\ Kapuściński, Murcia, Diego Marín, pp. 141-172.
}

36 Periodismohumano, $<$ https://periodismohumano.com>, 30 de diciembre de 2019.

37 Revista 5 W, <https://www.revista5w.com>, 30 de diciembre de 2019.

38 P. Simón, „Mujer, violencia y silencio”, Periodismohumano.com, 1.06.2011, <http://mujerviolenciaguatemala. periodismohumano.com/2011/06/01/el-primer-trabajo-con-ellas-es-que-nopiensen-que-es-normal-que-abusen-de-ellas>, 30 de diciembre de 2019. 
CARMONA JIMÉNEZ Javier

2009 "Narcohistorias de mujeres en Chile: Todo queda en familia", en: Santoro S., iSin nosotras se les acaba la fiesta! [América Latina en perspectiva de género], Bogotá, Centro de Competencia en Comunicación para América Latina, Friedrich Ebert Stiftung Artemisa Comunicación, pp. 15-24.

CASIQUE CASIQUE Leticia, FERREIRA FUREGATO Antonia

2006 "Violencia contra mujeres: reflexiones teóricas", Revista Latino-americana de Enfermagem, Ribeirão Preto, 14/6, Nov./Dec., en: SciELO, <https://www.scielo.br/pdf/ rlae/v14n6/es_v14n6a18.pdf $>$.

INSTITUTO OFICIAL DE RADIO Y TELEVISIÓN. RTVE

2002 Mujer, violencia y medios de comunicación, Madrid.

KAPUŚCIŃSKI Ryszard

2003 Los cinco sentidos de un periodista: (estar, ver, oír, compartir y pensar), México, Fondo de Cultura Económica.

KÖHLER Benedikt, SABRIA David, BLUMTRITT Jörg

2010 "The Slow Media Manifesto", <http://en.slow-media.net/manifesto>.

MATUD AZNAR María Pilar, FORTES MARICHAL Demelza, TORRADO MARTÍN-PALO-

MINO Esther, FORTES MARICHAL Saray

2018 Violencia contra las mujeres (Col. Cuadernos de Psicología 06), La Laguna (Tenerife), Latina.

LANDSBERG Alison

2004 Prosthetic Memory: The Transformation of American Remembrance in the Age of Mass Culture, New York, Columbia University Press.

LIZÁRRAGA Guadalupe

2017 Desaparecidas de la morgue, Editorial Casa Fuerte, Imperial Beach CA.

2017 "El periodismo independiente frente a la violencia en México", en: González-Esteban J.L., López-Rico C.M. (eds.), Periodismo, derechos humanos, migración y fronteras. Vigencia y legado de Ryszard Kapuściński, Murcia, Diego Marín, pp. 119-140.

LORENTE-ACOSTA Miguel

2003 "La violencia contra las mujeres en los medios de comunicación: entre la noticia y la información”, en: Ramírez Alvarado M. (coord.), Medios de comunicación y violencia contra las mujeres, Sevilla, Instituto Andaluz de la Mujer, Fundación Audiovisual de Andalucía, pp. 34-35.

MEJÍA MADRID Fabrizio

2012 "El teatro del crimen", en: Carrión J. (ed.), Mejor que ficción. Crónicas ejemplares, Barcelona, Anagrama, pp. 265-279.

ONU

1994 "Declaración sobre la Eliminación de la Violencia contra la Mujer", aprobada en Resolución de la Asamblea General de Naciones Unidas 48/104, del 20 de diciembre de 1993 publicada el 23 de febrero de 1994, en: <http://www.ohchr.org/SP/ProfessionalInterest/Pages/ ViolenceAgainstWomen.aspx>.

2006 "Fin a la violencia contra la mujer: Hechos, no palabras. Informe del Secretario General", $<$ https://www.un.org/es/events/endviolenceday/2006/pdf/v.a.w-exeS-use.pdf $>$.

PORTILLO Zoraida

2009 "Prensa chicha en Perú. Cuando las noticias se farandulizan", en: Santoro S., iSin nosotras se les acaba la fiesta! [América Latina en perspectiva de género], Bogotá, Centro de Competencia en Comunicación para América Latina, Friedrich Ebert Stiftung Artemisa Comunicación, pp. 65-74.

RIERA Carme

2019 "Violencia de género", La Vanguardia, 22.12.2019, <https://www.lavanguardia.com/opinion/20191222/472400291222/violencia-de-genero.html>. 


\section{RINCÓN Omar}

2009 "La narrativa en perspectiva de género" en: Santoro S., iSin nosotras se les acaba la fiesta! [América Latina en perspectiva de género], Bogotá, Centro de Competencia en Comunicación para América Latina, Friedrich Ebert Stiftung Artemisa Comunicación, pp. $5-10$.

RODRÍGUEZ WANGÜEMERT Carmen

2005 "Las crónicas: algunas ideas sobre la credibilidad en el periodismo interpretativo", Estudios sobre el mensaje periodístico, Universidad Complutense de Madrid, pp. 171-172.

RONQUILLO Víctor

2004 Las muertas de Juárez: Crónica de una larga pesadilla, Madrid, Temas de hoy.

ROSIQUE-CEDILLO Gloria, BARRANQUERO-CARRETERO Alejandro

2015 "Periodismo lento (slow journalism) en la era de la inmediatez. Experiencias en Iberoamérica", El Profesional de la Información, 24 (4):451, July 2015.

SALINAS MALDONADO Carlos

2019 "Elena Poniatowska: «Fue un abuso de un hombre adulto con una muchacha encandilada»", El País, 13.12.2019, <https://elpais.com/cultura/2019/12/13/actualidad/15762353 52_809332.html>.

SÁNCHEZ Gervasio

2001 "Guerras, mentiras y juegos de vídeo", en: Leguineche M., Sánchez G., Los ojos de la guerra, Barcelona, Debolsillo, pp. 461-472.

2019 "Violencias contra las mujeres", El Heraldo de Aragón, 17.12.2019, <https://www.heraldo.es/noticias/internacional/2019/12/17/violencias-contra-las-mujeres-exposicion-defotografias-de-gervasio-sanchez-1349321.html>.

\section{SANTORO Sonia}

2009 "Escribir lo que se nos de la gana", en: Santoro S., ;Sin nosotras se les acaba la fiesta! [América Latina en perspectiva de género], Bogotá, Centro de Competencia en Comunicación para América Latina, Friedrich Ebert Stiftung Artemisa Comunicación, pp. 11-14.

SILVA ORTEGA May

2003 "El papel de la educación y los medios de comunicación en la erradicación de la violencia contra las mujeres", en: Ramírez Alvarado M. (coord.), Medios de comunicación y violencia contra las mujeres, Sevilla, Instituto Andaluz de la Mujer, Fundación Audiovisual de Andalucía, pp. 24-25.

SIMÓN Patricia, BAULUZ Javier

2011 "Mujer, violencia y silencio", Periodismohumano.com, 1.06.2011, <http://mujerviolenciaguatemala.periodismohumano.com/2011/06/01/el-primer-trabajo-con-ellas-es-queno-piensen-que-es-normal-que-abusen-de-ellas $>$.

UN DEPARTMENT OF PUBLIC INFORMATION

2009 DPI/2546C, noviembre 2009, "Violencia contra las mujeres".

YUGUEROS GARCÍA Jesús

2014 "La violencia contra las mujeres: conceptos y causas", Barataria Revista CastellanoManchega de Ciencias Sociales, ${ }^{\circ} 18$. 


\section{Páginas de Internet}

Cosecha Roja, <http://cosecharoja.org/acerca-de/>.

Dromómanos, $<$ https://dromomanos.com>.

Periodismohumano, $<\mathrm{https}: / /$ periodismohumano.com/>.

Revista $5 W,<$ https://www.revista5w.com/>.

\section{Película}

ÁLVAREZ-STEHLE Chelo, Sands of silence, <http://www.sandsofsilence.org/anges-films-review-chelo-alvarez-stehles-sands-silence>.

\section{Violence against women through testimonies in journal reports}

Keywords: violence — woman — journalism — femicide — machismo - chronicles.

\section{Abstract}

The idea of the article is to present the problem of violence against women in contemporary Hispanic media. The study describes the concept and the way it is approached in journalism. It proves that journalists choose personal research and investigation in order to show the pain, suffering and death of women in different social contexts. Their work is often the only way to give testimony about the silenced problems.

Fecha de recepción: 22 de enero de 2020

Fecha de aceptación: 11 de mayo de 2020 